\title{
Cultural barriers in access to healthcare services for people with disability in Iran: A qualitative study
}

\author{
Shahin Soltani ${ }^{1}$, Amirhossein Takian ${ }^{1,2,3 *}$, Ali Akbari Sari ${ }^{1}$, Reza Majdzadeh ${ }^{4}$, Mohammad Kamali ${ }^{5}$
}

Received: 7 June 2016

Published: 3 Sep 2017

\begin{abstract}
Background: People with disability experience various problems to access to healthcare services. This study aimed to identify cultural barriers in access to healthcare services for people with disability in Iran.

Methods: We conducted a qualitative study using content analysis to identify the cultural barriers. We used semi-structured interviews to collect data. Participants were selected through purposeful sampling with maximum variation. 50 individual interviews were conducted with three groups of people with disability, healthcare services providers and policy makers, September to May 2015, at different locations in Tehran, Iran.

Results: We identified a number of different cultural barriers in access to health services for people with disability in Iran. These related to health service providers, namely reluctance to provide health services and disrespect; related to People with disability, namely denial of disability, disproportionate expectation, shame and insufficient sociocultural supports; and related to policy makers, namely lack of concern, little attention to the culture of disability and discrimination. We categorized misconception as a barrier that was observed at all levels of the society.

Conclusion: Disability is a reality that some human being may experience and live with it. The negative attitude towards people with disability has a close relationship with the cultural norms of a society. The culture of disability in different dimensions should be a priority for all policy makers. Removing cultural barriers in access to healthcare for people with a disability needs collective efforts and collaborations among all stakeholders.
\end{abstract}

Keywords: Disability, Access, Healthcare, Culture

Copyright@ Iran University of Medical Sciences

Cite this article as: Soltani Sh, Takian A, Akbari Sari A, Majdzadeh R, Kamali M. Cultural barriers in access to healthcare services for people with disability in Iran: A qualitative study. Med J Islam Repub Iran. 2017 (3 Sep);31:51. https://doi.org/10.14196/mjiri.31.51

\section{Introduction}

Access to healthcare is defined as the timely use of healthcare services to achieve the best health outcomes (1). People with disability (PWD) face more problems to access to healthcare compared to general population. Different economic, geographical, social and cultural factors can affect access to health services (2-5). Insurance coverage, income, cost of health services, information, transportation and communications have an important role to facilitate access to healthcare service for people with

\footnotetext{
Corresponding author: Dr Amirhossein Takian, takian@tums.ac.ir

1. Department of Health Management and Economics, School of Public Health, Tehran University of Medical Sciences, Tehran, Iran.

2. Department of Global Health and Public Policy, School of Public Health, Tehran University of Medical Sciences, Tehran, Iran.

3. Health Equity Research Centre (HERC), Tehran University of Medical Sciences, Tehran, Iran.

${ }^{4}$. Department of Epidemiology and Biostatistics, School of Public Health, and Knowledge Utilization Research Center, Tehran University of Medical Sciences, Tehran, Iran.

5. Department of Rehabilitation Management, School of Rehabilitation Sciences, Iran University of Medical Sciences, Tehran, Iran.
}

disability. Nevertheless, cultural aspects of accessibility to healthcare services are of triumph importance $(2,6,7)$.

Cultural factors can affect access to healthcare in various ways. Values and norms in a society restrict accessibility of healthcare for PWD (7). Various studies show that gender differences and racial discriminations can increase inequality in access to healthcare $(2,4,7)$. For example, some studies demonstrated that $\mathrm{w}$ omen with a disability were less likely than men with the same condi-

$\uparrow$ What is "already known" in this topic:

Society attitude toward people with disability is an important barrier in access to healthcare services.

\section{$\rightarrow$ What this article adds:}

This study show that cultural factors have an important role in hampering access to healthcare services among people with disability. These barriers include gender, education, health literacy and misconceptions. 
tion to use healthcare services (8-10). Another study showed that $5 \%$ of respondents had experienced some forms of discrimination during receiving health care (11). Also, some studies reported that racial and ethnic minorities were less likely than non-minorities to receive higher quality care services $(2,3,5,12)$.

According to the Anderson behavioral model, cultural factors are one of the most important predictors that can interact with other variables (13). In addition, Cultural differences may affect perceived needs and preferences of patients for health services. For example, Ling and Cheung found that people's ability to distinguish health problems is different within various cultures (14).

In Iran, evidence show that PWD faces more barriers to access to healthcare services compared with people without disability (15-18). The problems led to more sensitivity and attention towards disability among health policy makers to formulate policies to reduce such barriers. One of the most important policies was the Disability Protect Act passed in 2003 (19). The act provides legal protections for PWD in areas, i.e. access to public buildings, education, employment, inclusion, and finance. The act is currently under revision to address legal defects and create a comprehensive monitoring system to ensure compliance and clarity, taking culture of disability in the Iranian society into consideration.

Lack of attention to disability culture has led to different problems in access to health services for PWD in Iran. For example, the negative attitude was one of the barriers toin access to healthcare services for PWD in Iran (18). Another study found parents of children with an intellectual disability having a more negative attitude than parents of children without intellectual disability (20). To address the gap in the literature, this study aims to identify cultural factors affected access to healthcare services for PWD in Iran.

\section{Methods}

\section{Study design \& data collection}

We used semi-structured face-to-face interviews, using a generic interview guide developed specifically for this study (See Appendix), to collect data. Interviews were digitally recorded and transcribed verbatim. We provided participants an information sheet, explained the study for them and obtained their verbal consent, while reassuring them about the anonymity and confidentiality. The study was approved by the Ethical Committee of Tehran University of Medical Sciences (the ethical code: IR.TUMS.REC.1394.1794). All in all, we conducted 50 individual interviews, which lasted 30-90 minutes and held at different locations (interview with People with a disability was held in rehabilitation centers affiliated with the National Welfare Organization and the Iranian Red Crescent) from September 2015 until May 2016 in Tehran- Iran. The first author conducted the interviews, made brief notes during data collection and documented the date, time, place, events and other important parts of conversations. All authors involved in data analysis and final categorization of findings.

\section{Setting and sampling}

Our samples covered three groups: people with disability and their parents, healthcare services providers, and national policy makers. We chose participants using purposeful sampling method with maximum variation. Sampling strategy for PWD is showed in Table 1. For PWD who were unable to participate in the study due to cognitive impairments, we invited their parents to be interviewed. Health services providers who participated in our

Table 1. Sampling strategy for PWD selected for interview

\begin{tabular}{ll}
\hline Age & Ranged from 14-62 years old \\
\hline Gender & 10 women and 10 men \\
Location & From 7 rehabilitation centers across the megacity of Tehran \\
Participants' background & A mixture of Physical and intellectual disabilities, single and married, young and old, mild, \\
& middle and severe disability \\
Causes of disability (frequency) & $\begin{array}{l}\text { Spinal injury (3), cerebral palsy (3), intellectual disabilities (4), Multiple sclerosis (5), am- } \\
\text { putation (2), cerebrovascular accidents (1), physical injury (2) }\end{array}$ \\
\hline
\end{tabular}

Table 2. The characteristics of study participants

\begin{tabular}{|c|c|c|c|}
\hline & Type of participants & Male & Female \\
\hline \multirow[t]{2}{*}{ People with disability } & Physical disability & 7 & 9 \\
\hline & Intellectual disability & 3 & 1 \\
\hline \multirow[t]{7}{*}{ Health care providers } & Physician & & 1 \\
\hline & Psychologist & 3 & \\
\hline & Occupational therapist & 1 & \\
\hline & Caregiver & & 1 \\
\hline & Technical Orthopedist & 1 & \\
\hline & Manager & 1 & 1 \\
\hline & Nurse & & 1 \\
\hline \multirow[t]{9}{*}{ Policy makers } & Ministry of Health \& Medical Education (MOHME) & 4 & \\
\hline & State Welfare Organization of Iran & 4 & 1 \\
\hline & Social Security Organization & 2 & \\
\hline & Ministry of Cooperation, Labor and Social Welfare & 1 & \\
\hline & Foundation of Martyrs and Veterans Affairs & 1 & \\
\hline & Iran's Health Insurance Organization & 1 & 1 \\
\hline & Islamic Consultative Assembly & 1 & \\
\hline & NGOs & 2 & 1 \\
\hline & Tehran Municipality & 1 & \\
\hline Total & - & & \\
\hline
\end{tabular}


study had at least one year of work experience with people with disability. Interviews with providers were held in their work places. In addition, we interviewed national policy makers as well as some NGOs active in the field of disability. Table 2 summarizes the characteristics of study participants

\section{Data analysis}

We used the qualitative content analysis, using both deductive and inductive approaches for data analysis (21). Collecting and analysis of data were concurrent. After each interview, it was transcribed verbatim and then it was read several times for familiarization. In an inductive approach, we organized qualitative data in three steps, including open coding, creating categories and abstraction. All authors participated in interpretation of the coding tree. The first author conducted the open coding through identifying and naming the phenomena that were described in the text. For this, in line with the aims of the study, meaning units were extracted and then we put a label on each meaning unit named as a code The MAXQDA software program (version 11) was used to assist data storage and categorization. After open coding, codes were grouped into 10 categories. To reflect the main message of the interviews, codes were compared together and moved continuously between different categories. Categorization process was done by the first and second authors. Nevertheless, the whole steps were revised and approved by all authors. Finally, to enhance the credibility of our findings, we shared the transcripts, categories and subcategories with the participants to ensure the accuracy of our interpretations for the data. with participants (22, 23).

\section{Results}

Our main findings on cultural barriers to healthcare services are summarized in Table 3. Different barriers were reported by three groups of participants. Barriers related to healthcare service providers were reluctance to provide health services and disrespect. Barriers related to PWD were denial of disability, disproportionate expectation, shame and insufficient sociocultural supports. Finally, barriers related to policy makers were lack of concern, little attention to the culture of disability and discrimination. Misconception was a barrier that was observed across all levels of the society.

\section{Reluctance to provide health services}

One cultural issue that a number of people with disability raised was providers' reluctance to provide healthcare services for PWD. A woman with spinal cord injury said: "you must have a connection in a hospital in order to force them to do something for you; there must be someone there to force everyone to do you works unless you find someone very kind who helps you". Some interviewees complained that healthcare providers did not take the health problems of PWD particularly people with intellectual disability seriously and did not do their intervention with sufficient accuracy. One of the providers with a physical disability said "they say it is not worth it, even if we fill his teeth, he is still handicapped. Just one or two stitches are enough for him. There's no need to be careful; he isn't going to be married. They do not care much for these things". This low responsiveness sometimes led to more dangerous events. For example, one participant said: "our patient ended up in coma three times, no one would answer us, they said you must pay money ... we paid that money but he would become ill again, we said for god's sake, he is dying, but no one would listen".

\section{Disrespect}

Disrespect during provision of healthcare services was among barriers mentioned by people with a disability or their families. A lady with spinal cord injury who was using wheelchair said: "I went to a medical center, even though I was waiting in line, they said let's finish his work so we can get rid of him."

Another participant with physical problems complained from the negative attitude of healthcare providers towards people with disability: "For example, when someone with disability goes to a hospital they say 'they want to stay alive for what?' they don't treat that person so well "his parents will be more comfortable if he dies".

\section{Denial of disability}

A number of PWD and healthcare service providers

Table 3. Cultural barriers to access to healthcare services among PWD

\begin{tabular}{|c|c|c|c|}
\hline Theme & Category & Examples of cultural barriers & Relation \\
\hline \multirow[t]{12}{*}{$\begin{array}{l}\text { Cultural } \\
\text { factors }\end{array}$} & Reluctance to provide health services & $\begin{array}{l}\text { Dentists and physicians reluctance to provide health services for people with } \\
\text { disability }\end{array}$ & $\begin{array}{l}\text { Health services } \\
\text { provider (HSP) }\end{array}$ \\
\hline & Disrespect & To humiliate PWD when receiving health care & HSP \\
\hline & Denial of disability & Denying disability by families with PWD & PWD \\
\hline & Disproportionate expectations & Inappropriate expectations by PWD & PWD \\
\hline & Shame & $\begin{array}{l}\text { The shame feeling of women with disability to speak about their health prob- } \\
\text { lems }\end{array}$ & PWD \\
\hline & Insufficient sociocultural supports & Inadequate cultural supports by PWD's families & PWD \\
\hline & Lack of concern & $\begin{array}{l}\text { Lack of attention by health policy makers regarding health problems of per- } \\
\text { sons with disabilities }\end{array}$ & Policymakers \\
\hline & Little attention to cultural disability & $\begin{array}{l}\text { Little attention to the culture of disability in the media and the educational } \\
\text { system }\end{array}$ & Policymakers \\
\hline & Misconceptions & Assumptions that & Policy makers \\
\hline & & PWD are a cost burden & and \\
\hline & & PWD are not efficient & HSP/society \\
\hline & Discrimination & $\begin{array}{l}\text { Disability is limited to a special group, not the whole society } \\
\text { Discrimination between war-related disability and other groups of PWD }\end{array}$ & Policymakers \\
\hline
\end{tabular}


mentioned the fact that people who became disable due to car accidents needed longer time to cope with their disability. In addition, families of children with a mild intellectual disability did not accept their child's mental disability and therefore refused to go to medical centers. As a woman with physical disabilities said: "I myself was healthy and suddenly had to sit in a wheelchair. It's a very big shock. Think that you were healthy, you cloud study and go to school and suddenly you're sitting on a wheelchair or for example some of my friends are not still able to cope with this problem after 10 years and have severe depression. They stay at home and don't come out and are embarrassed when they sit on wheelchair".

One of the rehabilitation service providers said: "I now have a child who is 14 years old and his father has not yet accepted that his child has a problem".

Another one of health service providers who was providing rehabilitation services to people with intellectual disabilities expressed that unfortunately the golden time of treatment and rehabilitation is devoted to disputes and arguments in families:

"A lot of time passes but parents are arguing with each other. Father says you should be responsible for the child's problem and mother says no, your family had this problem and I didn't have anything to do with it and my family had no problem".

\section{Disproportionate expectations}

Some policy makers and health service providers stated that many PWD expected the National Welfare Organization (NWO) to cover their needs, whereas the organization alone was unable to address all these demands. Some interviewees considered the Ministry of Health and Medical Education (MOHME) as the main responsible body for healthcare services, while the NWO was expected to act consistently with the MOHME in solving some of the health problems. One participant noted "of course to avoid unilateral judge, we must say that some families want anything from welfare and say because our child is under the cover of welfare, they must do everything for him."

Also, a policy maker said: "Unfortunately, people with disability feel that an organization such as the welfare should be responsible for all of their needs and as said by Dr. ... disabled person refers to National Welfare Organization even for a simple cold".

\section{Shame}

Some women participants believed that women with disabilities faced a lot of problems with pregnancy, menstrual and sexual problems. Worse still, their referral to higher level of healthcare was problematic, as it was said by a woman with physical disability: "A disabled woman is not like a normal woman and has is ashamed of herself because of having these problems. A woman with spinal cord injury has a lot of problems due to her conditions and has a different condition compared to a normal person and cannot easily refer to any doctor".

The study participants also stated that lack of expressing these problems may exacerbate their health problems. Hence they might face other numerous complications in the future. A health service provider said: “They don't say their issues to the doctor, maybe because they feel ashamed to talk about such issues with the doctor and this will later lead to other problems which cannot be solved easily."

Lack of expression will not only lead to further personal complications, but it can also affect the marital relationship as well as their family, as mentioned by a health services provider: "There were even some people here who suffered from cervical cancer just because they didn't tell their problem to anyone before this and they even had to get divorced".

\section{Insufficient support of families}

One problem that was frequently raised by health service providers was the cultural problems in the families of PWD who were from low socioeconomic background, hence they received poor social and financial support from their families. The insufficient family support affected the treatment and rehabilitation process of PWD. One rehabilitation service provider described the interview that he had with a mother who had a child with intellectual disability: "after 5 or 6 months that the child is coming here, his mother's swearing that she's working so she can pay the costs because the child's father has said I won't pay a penny for him to come here, you see how low level some families are".

Another rehabilitation service provider also complained from the low level of interaction with rehabilitation centers by families coming from lower socioeconomic levels: "I have to force the families to come here so that I can speak to them about something; the level of interaction is low."

Being in lower socioeconomic families was perceived to have negative effects on physical and mental health of children with disability. A rehabilitation service provider expressed his experience in this regard: "for example, I have told a family that your child has broken the glass with his fist so it is obvious that he should be under supervision of a psychiatrist and they answer that we won't take him and I ask why, and they answer that we give him some medicines that will force him just to lay down and not be able to do anything!".

In contrast, families with upper socioeconomic status provided greater support for their children with disability and created continuous and appropriate communication with rehabilitation centers: "families with higher intellectual, cultural and social levels have better cooperation, we have families that come to the meetings, have continuous presence, are consistently following up and we have no problem with them".

\section{Lack of concern among health policy makers}

A number of PWD were concerned about the lack of sympathy among officials and policy makers towards the challenges of people with disability, this leads to a lack of prioritization of the issues of PWD by policymakers. As mentioned by a policy maker in a non-governmental organization (NGO): "Unfortunately, most authorities know the problem and the issue, but they do not care be- 
cause it does not matter to them."

A policymaker who had physical disability himself said that authorities are solely looking for the media and promotional activities and do not pay much attention to the health needs of the disabled minority: "Authorities don't like to spend their time on these things and advertising and media matter most to them."

\section{Little attention to the culture of disability}

Some people with disability pointed out that the rest of population are still unaware of the culture of disability and appropriate ways of treating a person with a disability. There was a consensus that such a problem has jeopardized PWD's access to health services. In addition, participants noted the important role of the media in the culture of disability and increasing people's knowledge about disability: "The media is the most important tool these days. The media is very pervasive because people spend much of their time with media. The media must spend more time and money on this issue to make people more familiar with disables and disabilities". Our participants also stated that lack of enough attention to disability might lead to adverse effects on people's views toward people with disability in the society.

\section{Misconception}

People with disability and some policymakers complained about misconceptions among some healthcare policy makers about PWD and the need to change such views and beliefs. Some interviewees were of the view that spending money for PWD will affect their life and for this reason, the policymakers did not want to spend meaningful money on such aspects. One policy maker from an NGO said: "They think that disabled people are costly, so if they want to change their attitude this must cost a lot always... They think people with disability are not productive and productive, so they are a burden on them".

Some interviewees asked the policymakers to change their attitudes towards disability and consider it as a public issue and not just a problem of a specific group: "All policymakers should change their attitude toward disability and consider it as a public problem for the whole community rather than PWD themselves".

In addition, some interviewees pointed out the existence of negative judgments and lack of respect towards PWD in the society. One healthcare services provider stated his experience in this field: "it is interesting that our neighbor always said that these children should never be even allowed to live in the town and I replied where they should live then? Take them out of the city and rent a garden for them, he stated".

\section{Discrimination}

Discrimination among different groups of PWD by government officials and policymakers was perceived to have affected the access of PWD to healthcare services. It was mentioned that people who have disabilities due to wars and military conflict can enjoy free use of health services almost always freely, whereas people with genetic and congenital disabilities or injuries must pay for health costs similar to other people. One policy maker with a physical disability said: "the definitions of veterans and disabled are very different in countries like ours. Disability definition for a person who is $96 \%$ disabled is different with a person who became disabled during war with $25 \%$ disability ratio. Of course, it is true that these people were protecting the sovereignty of Iran when they became disabled. However, other disabled people became disabled unwantedly and don't want to take advantage from their condition therefore. Disability is not a condition that anyone wants to have and it is not in a way that someone would enjoy it".

\section{Discussion}

This study aimed to identify cultural barriers affecting access to healthcare services for people with disability in Iran. Our results indicate that in spite of the passage of Disability Product Act in 2003 in Iran, significant cultural barriers still exist in access to healthcare services for PWD. These barriers are scattered across various layers of the society and can limit access to healthcare services in various forms. Experiences of PWD in some healthcare environments, e.g. hospitals, emergency rooms, and medical clinics showed that some service providers might undermine the dignity and value of PWD and consider the provision of healthcare services for PWD as a time- consuming and frustrating work. Hence, providers may be reluctant to provide healthcare services because they might feel PWD are an additional burden for them.

Studies show that applying communication and assistive technologies for PWD have positive effects on patient provider relationships (24-27). If healthcare facilities, i.e. hospitals employ more modern technologies for communication, treat and transport PWD, the willingness of their staff might increase to provide health services for PWD. Most PWD reported their lack of satisfaction with the provided health services culturally (28). Another Study underpinned the lack of providers' willingness as an impediment to receive rehabilitation services (18).

According to the World Health Organization's (WHO) framework of 2000 for improving health systems performance in member states (29), health systems need to embrace mechanisms to improve their responsiveness. PWD expect to be treated with dignity. Poor responsiveness can reduce the utilization of and access to healthcare services by PWD (30). This renders appropriate planning to develop providers' knowledge about the health needs of PWD, perhaps by holding training courses of different forms to reduce problems (31).

Denial of disability, shame, fear and distrust, inappropriate expectations and insufficient supports were other cultural problems observed within PWD and their families. Other studies have related the denial of disability with poor psychological judgments $(32,33)$. In addition to sociocultural factors, such judgments may be related to variables such as age, gender, and literacy. The duration between the beginning of disability and acceptance may take several years. The denial of disability can affect motivation, participation and treatment outcomes (34). Therefore, easier access to psychological services should 
be created for PWD and their families to help facilitate the rapid acceptance of disability and integration of PWD within communities $(35,36)$.

This study revealed fear and distrust towards the health system rooted in cultural aspects of the society. We found two causes of distrust in the health system among PWD. First, insufficient knowledge of healthcare providers towards the health needs of PWD, as observed in other studies $(37,38)$, which can be addressed by holding specific training courses for health service providers. Second, PWD did not trust the health insurance coverage to reimburse their health expenditures. They mentioned that the cost of diagnostic tests, assistive technologies, and medicines were very high and unaffordable and decreased access to health care for PWD in Iran (39). Other studies showed the lack of trust in the health system associated with low self-reporting of health problems. Previous experiences of PWD with the healthcare system have a significant effect on their views towards healthcare, i.e. AfricanAmericans' fear of being treated as guinea pigs in clinical researches (40).

The inappropriate expectation of PWD to receive all kinds of health services from the National Welfare Organization affected their access to healthcare services. Such expectations may arise from inadequate access to health information, which have a determinant role in access to health care (41). The media, i.e. radio, television, newspapers and social networks are good sources to enhance awareness and to reduce inequalities in access to healthcare services. Access to timely, accurate and comprehensive information about different types of health service providers, can increase PWD's awareness about the performance of health system (42). Although the NWO formulates rehabilitation policies and is in charge of providing some rehabilitation and care services to PWD in Iran, it is unable to meet the entire health needs of PWD on its own. As the steward of the health system, the Ministry of Health and Medical Education is expected to take a more active play in providing healthcare services for PWD and increase their access to health services in Iran.

Sociocultural status of PWD and their families may affect their level of support. Cultural status can influence people's perception of health and disability (43), or cultural beliefs can change treatment alternatives (43-45). The relationship between disability and poverty has been studied $(46,47)$. Disability can be a cause or consequence of poverty. PWD are more likely to live in poverty compared to the general population (48). Governments should formulate supportive policies to reduce financial problems of PWD and their families. As long as PWD and their families experience financial problems, they cannot think of cultural issues and other needs of PWD.

Lack of concern among health policy makers, misconception and provision of privilege for war-related PWD to access to healthcare services free of charge, while other PWD must bear the costs of healthcare like other citizens, was criticized by our participants. It is expected the government attempts to increase its commitment to galvanize the rights of PWD and their families. Revision of disability conceptions, classification systems, and legal rulings can change public policies for PWD as a priority for informed policy makers (49).

\section{Conclusion}

Cultural accessibility to health services is crucial because the meaning of disability and health in a country might be different from another. The culture of disability in various dimensions should be a priority for policy makers, addressing that may require collective efforts and collaborations among all informed stakeholders. One effective way to improve this is the greater engagement of PWD during policy-making process. The negative attitude towards PWD has a close relationship with a culture of society. Thus formulation and implementation of public policies to promote the culture of disability should be put on the top agenda of policy makers.

\section{Limitations}

This study had two main limitations. First, people with vision and hearing disabilities were not among participants of this study, whose specific problems to access to healthcare services should be addressed in the future studies. Second, some participants were not able to participate in the verification process either because of unavailability (some busy policy makers and services providers) or being illiterate (some PWD or their parents).

\section{Acknowledgments}

The authors would like to express their deepest gratitude to all stakeholders, health service providers, people with disability and their families who participated in this study, which was funded by the Tehran University of Medical Sciences (TUMS).

\section{Conflict of Interests}

The authors declare that they have no competing interests.

\section{References}

1. Gulliford M, Figueroa-Munoz J, Morgan M, Hughes D, Gibson B, Beech R, et al. What does' access to health care'mean? J Health Serv Res Policy. 2002;7(3):186-8.

2. Ashton CM, Haidet P, Paterniti DA, Collins TC, Gordon HS, O'Malley K, et al. Racial and ethnic disparities in the use of health services. J Gen Int Med. 2003;18(2):146-52.

3. Baru R, Acharya A, Acharya S, Kumar AS, Nagaraj K. Inequities in access to health services in India: caste, class and region. Econom Politi Weekly. 2010:49-58.

4. Hazarika G. Gender differences in children's nutrition and access to health care in Pakistan. J Develop Stud. 2000;37(1):73-92.

5. Wilson PA, Yoshikawa H. Improving access to health care among African-American, Asian and Pacific Islander, and Latino lesbian, gay, and bisexual populations: Springer; 2007.

6. Betancourt JR, Green AR, Carrillo JE, Ananeh-Firempong 2nd O. Defining cultural competence: a practical framework for addressing racial/ethnic disparities in health and health care. Pub Health Report. 2003;118(4):293.

7. Brach C, Fraserirector I. Can cultural competency reduce racial and ethnic health disparities? A review and conceptual model. Med Care Resh Rev. 2000;57(4 suppl):181-217.

8. Wei W, Findley PA, Sambamoorthi U. Disability and receipt of clinical preventive services among women. Women's Health Issu. 2006;16(6):286-96.

9. Merten JW, Pomeranz JL, King JL, Moorhouse M, Wynn RD. Barriers to cancer screening for people with disabilities: A literature 
review. Disab Health J. 2015;8(1):9-16.

10. Diab ME, Johnston MV. Relationships between level of disability and receipt of preventive health services. ACRM. 2004;85(5):749-57.

11. Trivedi AN, Ayanian JZ. Perceived discrimination and use of preventive health services. J Gen Int Med. 2006;21(6):553-8.

12. Egede LE. Race, ethnicity, culture, and disparities in health care. J Gen Int Med. 2006;21(6):667-9.

13. Andersen RM. Revisiting the behavioral model and access to medical care: does it matter? Journal of health and social behavior. 1995:1-10.

14. Lin KM, Cheung F. Mental health issues for Asian Americans. Psychiatric Serv. 1999.

15. Sajedi F, Alizad V, Malekkhosravi G, Karimlou M, Vameghi R. Depression in mothers of children with cerebral palsy and its relation to severity and type of cerebral palsy. Acta Medica Iranica. 2010;48(4):250.

16. Raeis-Dana M, Tabatabaei-Nia M, Kamali M, Shafaroudi N. From diagnosis to coping: a journey with parents in the course of the disability of their children. Journal of Rehabilitation. 2009;10(1):42 51.

17. Akbari R, Kamali M, Ashayeri H, Shafaroodi N. Challenge of Private Rehabilitation Centers and Welfare Organization (Behzisti). Iranian Rehabilitation J. 2011;9(14):37-44.

18. Abdi K, Arab M, Rashidian A, Kamali M, Khankeh HR, Farahani FK. Exploring barriers of the health system to rehabilitation services for people with disabilities in Iran: A Qualitative Study. Electronic Phys. 2015;7(7):1476.

19. Bahreini R, editor Understanding Disability as a Human Rights Issue. Gozaar: A Forum on Human Rights and Democracy in Iran Message posted to http://www gozaar org/english/articlesen/Understanding-Disability-as-a-Human-Rights-Issue html; 2007.

20. Mobaraki A. Comaparision of knowledge and attitude of mothers with mentally retarded children with those without mentally retarded children in Gachsaran (2003). Armaghane Danesh. 2003;8(3):81-90.

21. Elo S, Kyngas H. The qualitative content analysis process. Journal of advanced nursing. 2008;62(1):107-15.

22. Hagens V, Dobrow MJ, Chafe R. Interviewee Transcript Review: assessing the impact on qualitative research. BMC Med Res Method. 2009;9(1): 1 .

23. Goldblatt H, Karnieli-Miller O, Neumann M. Sharing qualitative research findings with participants: Study experiences of methodological and ethical dilemmas. Patient Educ Counsel. 2011;82(3):389-95.

24. French S, Swain J. Disability and communication: Listening is not enough. Communication, relationships and care: A reader. 2004:220.

25. Hemsley B, Balandin S, Worrall L. The "Big 5" and beyond: Nurses, paid carers, and adults with developmental disability discuss communication needs in hospital. Appl Nurs Res. 2011;24(4):e51-e8.

26. Lupton D, Seymour W. 'I am Normal on the 'Net': Disability, Computerised Communication Technologies and the Embodied Self. In: Coupland J, Gwyn R, editors. Discourse, the Body, and Identity. London: Palgrave Macmillan UK; 2003. p. 246-65.

27. Ripat J, Woodgate R. The intersection of culture, disability and assistive technology. Disabil Rehabil Assist Technol. 2011;6(2):87-96.

28. Zarei Matin H, Jandaghi G, Heydari F, Imani M. The Attitude of War Handicaped toward the Services Recieved in Terms of their needs. Iran J War Public Health. 2010;2(2):22-31.

29. Organization WH. The world health report 2000: health systems: improving performance: World Health Organization; 2000.

30. Atun R, Jaffar S, Nishtar S, Knaul FM, Barreto ML, Nyirenda M, et al. Improving responsiveness of health systems to non-communicable diseases. The Lancet. 2013 Feb 23;381(9867):690-7.

31. Valentine NB, de Silva A, Kawabata K, Darby C, Murray CJ, Evans $\mathrm{DB}$, et al. Health system responsiveness: concepts, domains and operationalization. Health systems performance assessment: debates, methods and empiricism. 2003:573-96.

32. Olney MF, Brockelman KF, Kennedy J, Newsom MA. Do you have a disability? A population-based test of acceptance, denial, and adjustment among adults with disabilities in the US. J Rehabil. 2004;70(1):4.

33. Ali A, King M, Strydom A, Hassiotis A. Self-reported stigma and its association with socio-demographic factors and physical disability in people with intellectual disabilities: results from a cross-sectional study in England. Soc Psychiatry and Psychiatr Epidemiol. 2015:1-10.

34. Katz N, Fleming J, Keren N, Lightbody S, Hartman-Maeir A Unawareness and/or denial of disability: Implications for occupational

therapy intervention. Can J Occup Therapy. 2002;69(5):281-92.

35. Lutz BJ, Bowers BJ. Disability in everyday life. Qualitat Health Res. 2005;15(8):1037-54.

36. Kamali M, Chabok A. Rehabilitation Needs of People with Cerebral Palsy: a qualitative Study. MJIRI. 2014;28:16.

37. Drainoni ML, Lee-Hood E, Tobias C, Bachman SS, Andrew J, Maisels L. Cross-disability experiences of barriers to health-care access consumer perspectives. J Disabil Pol Stud. 2006;17(2):101-15.

38. Vameghi R HBM, Hatami-Zadeh N, Biglarian A, Rah-Chamani M R. Factors Affecting Delayed Referral for Speech Therapy in Iranian children with Speech and Language Disorder. J Rehabil. 2014;14(6):68-77.

39. Abolhallaje M, Hasani S, Bastani P, Ramezanian M, Kazemian M. Determinants of catastrophic health expenditure in iran. Iran J Pub Health. 2013;42(1):155

40. Kennedy BR, Mathis CC, Woods AK. African Americans and their distrust of the health care system: healthcare for diverse populations. J Cultur diver. 2007;14(2):56-60.

41. Thiede M. Information and access to health care: is there a role for trust? Soc Sci Med. 2005;61(7):1452-62.

42. Rajati F, Kamali K, Parvizy S. Public Health Customers' Experiences of Health Accessibility: A Phenomenological Study. Iran J Epidemiol. 2011;7(2):17-24.

43. Ravindran N, Myers BJ. Cultural influences on perceptions of health, illness, and disability: A review and focus on autism. J Child Fam Stud. 2012;21(2):311-9.

44. Kleinman A, Good B. Culture and depression. New Eng J Med. 2004;351:951-2.

45. Flores G. Culture and the patient-physician relationship: achieving cultural competency in health care. J Pediatri. 2000;136(1):14-23.

46. Mitra S, Posarac A, Vick B. Disability and Poverty in Developing Countries: A Multidimensional Study. World Dev. 2013;41:1-18.

47. Palmer M. Disability and poverty: A conceptual review. J Disabil Policy Stud. 2011;21(4):210-8.

48. Braithwaite J, Mont D. Disability and poverty: a survey of World Bank poverty assessments and implications. ALTER-Europ J Disabil Res. 2009;3(3):219-32.

49. Shogren KA, Bradley VJ, Gomez SC, Yeager MH, Schalock RL, Borthwick-Duffy $\mathrm{S}$, et al. Public policy and the enhancement of desired outcomes for persons with intellectual disability. Intellect Dev Disabil. 2009;47(4):307-19.

\section{Appendix A}

Interview guide

People with disability and health services providers

1. Do you/ people with disability have all the support that you/they need from your/ their family and friends? Please discuss

2. Do you/ people with disability feel that people in the community judge you/ them negatively for attending this facility? Please discuss

3. In this clinic are you/ people with disability able to talk to the doctors or nurses in private? Please discuss

4. Could health services providers speak languages you/ people with disability prefer? Please discuss

5. Is Patient information kept confidential in this clinic? Please discuss

6 . Do health workers treat you/ people with disability with sufficient respect? Please discuss

7. Do doctors and nurses (health workers) discuss the treatment fully with you/ people with disability? Please discuss

Policy makers and health services providers

8. In your opinion, are cultural problems effective to decrease access to health care for people with disability? How?

9. Would you please, give your opinion about the role of policy makers to decrease cultural problems?

10. What policies have been adopted to increase cultural access to healthcare?

11. Please, explain the strengths and weaknesses of the policies?

12. What is your opinion about implementation of these policies?

13. What were the effects of the policies on cultural access to healthcare?

14. As a final question, is there any further issue you would like to add? 